\title{
Dentsply Sirona übernimmt MIS Implants
}

Dentsply Sirona hat bekannt gegeben, die Aktienmehrheit am Implantathersteller MIS Implants Technologies Ltd. aus Barlev, Israel, zu übernehmen. Die Übernahme von MIS Implants durch Dentsply Sirona eröffnet beiden Unternehmen neue Wachstumsperspektiven, von denen Kunden und Patienten weltweit profitieren MAKE IT SIMPLE tatmarkt zu bedie- $\begin{aligned} & \text { werden. „MIS ist per- } \\ & \text { fekt aufgestellt, um } \\ & \text { das Value-Segment } \\ & \text { im heimischen und } \\ & \text { weltweiten Implan- }\end{aligned}$ nen. Aus strategischer Sicht ist es wichtig, sowohl dieses als auch das PremiumSegment mit einem breiten Spektrum an Implantatmarken abzudecken. Dentsply Sirona möchte für jede Indikation und jeden Patienten- bzw. Behandlerwunsch ein passendes Produkt anbieten können. In dieser Hinsicht ergänzt MIS Implants das Produktportfolio von Dentsply Sirona optimal“, so Jeffrey T. Slovin, Chief Executive Officer von Dentsply Sirona..

Nach einer Pressemitteilung von

Dentsply Sirona, USA - York

www.dentsplysirona.com 